\title{
Are children and adolescents with cystic fibrosis at lower risk of caries?
}

\author{
Abstracted from \\ Chi DL.
}

Dental caries prevalence in children and adolescents with cystic fibrosis: a qualitative systematic review and recommendations for future research. Int J Paediatr Dent 2013; 23: 376-386. doi: 10.1111/ipd.12042. Epub 2013 Jun 12. PubMed PMID: 23758751; PubMed Central PMCID: PMC3745806.

Address for correspondence: Donald L. Chi, DDS, PhD, Department of Oral Health Sciences, University of Washington, Box 357475, Seattle, WA 98195, USA. E-mail: dchi@uw.edu

\section{Question: What is the prevalence of caries in children with cystic fibrosis (CF)?}

Data sources The Medline, Embase and Web of Science databases were searched.

Study selection Human clinical trials, evaluation studies and systematic reviews related to dental caries prevalence in children and adolescents with CF under age 18 years were included.

Data extraction and synthesis Standard date items were extracted and risk of bias was assessed using adapted criteria from the Preferred Reporting Items for Systematic reviews and Meta-Analyses (PRISMA) statement. A qualitative synthesis was conducted.

Results Fifteen studies met the inclusion criteria, 12 studies were considered as being at high risk of bias and three as medium risk. Ten studies concluded that children with CF had significantly lower caries prevalence than control children, while three studies reported that children with CF had higher caries prevalence, and two studies found no difference by CF status. All of the studies had limitations that may bias the results.

Conclusions While children with CF may be at lower risk for dental caries, adolescents with CF may not be at lower caries than those without CF. Additional research is needed to evaluate a potentially flawed paradigm regarding caries risk in children and adolescents with CF.

\section{Commentary}

Cystic fibrosis is a genetic disease that leads to overproduction and accumulation of mucus in the lungs, with can result in airway obstruction and life-threatening bacterial infections.

Cystic fibrosis is most common in white people of northern European descent. Within the UK the prevalence of cystic fibrosis is approximately 1 in 2,500 newborns and approximately 9,000 people in the UK live with the condition. The prognosis for people with cystic fibrosis has improved due to advancements in treatment, though there is still no cure, so dental care professionals should be aware of potential risk factors and how to treat patients with cystic fibrosis.

The effect of cystic fibrosis on the oral cavity has been of interest to researchers for many years but has resulted in contrasting conclusions, which may cause confusion when planning oral care for patients with cystic fibrosis. Therefore, this review set out to determine whether children and adolescents with cystic fibrosis are at higher or lower risk of developing dental caries. The Dental Elf commented on this review in June 2013 (http://www.thedentalelf. net/2013/06/17/limited-evidence-available-of-the-caries-risk-inchildren-and-adolescents-with-cystic-fibrosis/). A single author conducted this qualitative systematic review and intimated this could have led to bias - one could question why the author did not engage with others to carry out a joint review to strengthen the review process rather than identify this as a risk in the paper.

A fairly comprehensive literature search was carried out and included studies of different designs (human clinical trials, evaluation studies and systematic reviews), exploring three online databases. The studies were limited to those written in English and you could argue that selecting only English language papers was satisfactory since cystic fibrosis is more common in the northern European population, but there was no mention of the grey literature.

The search covered a period of 53 years, which could potentially influence the findings, as the treatment of cystic fibrosis, and also the prevention and management of dental caries has advanced significantly over this time. One example, found in two of the older papers, mentioned the higher incidence of tooth discolouration and hypoplastic defects of the permanent teeth in patients with cystic fibrosis due to tetracyclines. This should be a problem of the past as the antibiotic of choice has changed in more recent years. 
The aim of a qualitative systematic review is to collate and summarise the literature to establish whether there are trends in the findings, and identify potential sources of bias which could help design more robust studies with a reduced level of bias in the future.

An adapted version of PRISMA (Preferred Reporting Items for Systematic reviews and Meta-Analyses) statement assessed the risk of bias. The author clearly explains the summary measures used to assess study bias and highlights some of the problems as to why it is hard to come to any definite conclusions. None of the studies with control groups provided clear rationale for having a control group, less than half of the included studies justified the statistical approach taken, only one study documented there was more than one dental examiner, not one study specified whether the examiner(s) were blinded and fewer than half the studies included details on the assessment of intra- or inter-rater reliability. Hence, of the 15 studies that met the inclusion criteria, twelve were classified as high risk of bias, three as medium and no studies were identified to be of low risk.

Theoretically, cystic fibrosis patients could be considered as high caries risk as they routinely consume high sugar diets involving the need for high frequency intake. They may also have additional risks relating to regular medications including syrupy suspensions and aerosol inhalers, which are also acidic with a low initial $\mathrm{pH}$. There are also studies that report a reduction in salivary flow, increased Streptococcus mutans levels and patients suffering from oesphageal reflux as well as mouth breathing caused by nasal and sinus obstructions.

On the contrary, other studies suggest that patients with cystic fibrosis have lower Streptococcus mutans counts than control groups due to the long-term use of antibiotics, although some contradict this finding and ask for further studies to be carried out to explore if there is a salivary mechanism that helps. Observations also suggest there is less dental plaque and gingivitis, possibly related to the long-term use of antibiotics. Candida infections are rare despite the long-term use of antibiotics but cystic fibrosis patients should not be considered immune deficient.

The review set out to explore the prevalence of caries in children and adolescents under 18 years old. However, some studies were included that had adults over the age of 18 years old which were not able to be separated from the age group the reviewas interested in, leading to possible skewing of the results. The five studies involving patients over 18 years old reported different results, with one reporting a higher caries incidence rate and others 'a significantly reduced caries experience' or 'did not have significantly more dental decay'. Therefore, there is a lack of clear evidence to determine if children and adolescents with cystic fibrosis are at higher risk or lower risk for dental caries. These contrasting findings make it hard to draw conclusions to help understand the nature and extent of oral health problems in patients with cystic fibrosis to help plan oral health services to meet the needs of children and adolescents.

From a practice perspective it is important to risk assess each patient individually, taking into consideration the wider determinants of health as well as the condition and treatment plan accordingly, ensuring that oral health promotion, prevention and preventive care is managed appropriately.

In summary, this qualitative systematic review found studies of a high or medium risk of bias with conflicting outcomes when considering the dental caries prevalence of children and adolescents with cystic fibrosis. Further research is required to explore the effect of cystic fibrosis on oral health. Based on these findings and potential complications in treating patients with cystic fibrosis it is advisable to raise awareness amongst general dental practitioners and encourage the implementation of a preventive programme from birth to reduce the need for treatment, including routine professional care and promoting the need for very good oral hygiene habits at home.

\section{Practice points}

- It is important to work as part of a multi-disciplinary team and be aware of possible risks to oral health (high frequency and high calorie diets) and also potential positive effects on oral health such as the long-term use of antibiotics.

- Patients with cystic fibrosis require routine dental care similar to that of general population.

- Prevention is better than cure for both oral health and general health, therefore it is important to stress the key oral health messages, especially during the transition from childhood to adolescent.

\section{Emma 0'Keefe}

Department of Public Health, NHS Fife, Leven, Scotland, UK

Evidence-Based Dentistry (2014) 15,46-47. doi: 10.1038/sj.ebd.6401024 